OPEN ACCESS

Edited by:

Qiyu Sun,

University of Central Florida,

United States

Reviewed by:

Bei Liu,

Tianjin University of Science and

Technology, China

Ernesto De Vito,

Università di Genova, Italy

${ }^{*}$ Correspondence:

Roza Aceska

raceska@bsu.edu

Specialty section:

This article was submitted to Mathematics of Computation and

Data Science,

a section of the journal

Frontiers in Applied Mathematics and

Statistics

Received: 31 August 2017

Accepted: 24 October 2017

Published: 07 November 2017

Citation:

Aceska R and Kim YH (2017) Scalability of Frames Generated by

Dynamical Operators.

Front. Appl. Math. Stat. 3:22

doi: 10.3389/fams.2017.00022

\section{Scalability of Frames Generated by Dynamical Operators}

\author{
Roza Aceska ${ }^{1 *}$ and Yeon H. Kim ${ }^{2}$ \\ ${ }^{1}$ Department of Mathematical Sciences, Ball State University, Muncie, IN, United States, ${ }^{2}$ Department of Mathematics, \\ Central Michigan University, Mount Pleasant, MI, United States
}

Let $\mathbb{H}$ be a separable Hilbert space, let $G \subset \mathbb{H}$, and let $A$ be an operator on $\mathbb{H}$. Under appropriate conditions on $A$ and $G$, it is known that the set of iterations $F_{G}(A)=\left\{A^{j} g \mid g \in\right.$ $G, 0 \leq j \leq L(g)\}$ is a frame for $\mathbb{H}$. We call $F_{G}(A)$ a dynamical frame for $\mathbb{H}$, and explore further its properties; in particular, we show that the canonical dual frame of $F_{G}(A)$ also has an iterative set structure. We explore the relations between the operator $A$, the set $G$ and the number of iterations $L$ which ensure that the system $F_{G}(A)$ is a scalable frame. We give a general statement on frame scalability, and study in detail the case when $A$ is a normal operator, utilizing the unitary diagonalization. In addition, we answer the question of when $F_{G}(A)$ is a scalable frame in several special cases involving block-diagonal and companion operators.

Keywords: dynamical sampling, frames, scalable frames, iterative actions of operators

\section{INTRODUCTION}

The problem of generating frames by iterative actions of operators [1-3] has emerged within the research related to the dynamical sampling problem $[1,3-6]$. The conditions under which a frame generated by iterative actions of operators exists for a finite-dimensional or a separable Hilbert space have been stated in Aldroubi et al. [1,3]. If we have a frame, then a linear combination of a dual frame with the dynamically sampled coefficients reproduce the original signal. The natural follow-up questions to ask in this setup are: whether we can obtain a scalable [7-13] frame under iterative actions, and if not, whether we can find a dual frame which preserves the dynamical structure.

Let $A$ be an operator on a separable Hilbert space $\mathbb{H}$. We consider a countable set of vectors $G$ in $\mathbb{H}$, and a function $L: G \rightarrow \mathbb{N}_{0}$, where $\mathbb{N}_{0}=\mathbb{N} \cup\{0\}$. Related to the iterated system of vectors

$$
F_{A}^{L}(G):=\left\{A^{j} \mathbf{g} \mid \mathbf{g} \in G, 0 \leq j \leq L(\mathbf{g})\right\},
$$

we answer the following two questions:

(Q1) For which matrices $A$, which sets $G$, and which limits of iterations $L$ is system (Equation 1 ) a scalable frame for $\mathbb{H}$ ?

(Q2) Assuming that system (Equation 1) is a frame for $\mathbb{H}$, can we obtain a dual frame for Equation

(1), perhaps by iterative actions of some operator?

The motivation for studying systems of type (Equation 1) comes from the dynamical sampling problem (DSP): Let the initial state of a dynamical system be represented by an unknown element $\mathbf{f} \in \mathbb{H}$. Say the initial state $\mathbf{f}$ is evolving under the action of an operator $A^{*}$ to the states $\mathbf{f}_{j}=A^{*} \mathbf{f}_{j-1}$, where $\mathbf{f}_{0}=\mathbf{f}$ and $j \in \mathbb{N}_{0}^{+}$. Given a set of vectors $G \subset \mathbb{H}$, one can find conditions on $A, G$ and 
$L=L(\mathbf{g})$ which allow the recovery of the initial state $\mathbf{f}$ from the set of samples $\left\{\left\langle A^{* j} \mathbf{f}, \mathbf{g}\right\rangle \mid \mathbf{g} \in G\right\}_{j=0}^{L(\mathbf{g})}$. In short, the problem of signal recovery via dynamical sampling is solvable if the set of vectors (Equation 1) is a frame for $\mathbb{H},[1,3]$. In frame theory it is known that every frame has at least one dual frame; if $F_{A}^{L}(G)$ is a frame for $\mathbb{H}$, and its dual frame elements are $\mathbf{h}_{\mathbf{g}, j}$, then all $\mathbf{f} \in \mathbb{H}$ are reconstructed as

$$
\mathbf{f}=\sum_{\mathbf{g} \in G} \sum_{j=0}^{L(\mathbf{g})}\left\langle\mathbf{f}, A^{j} \mathbf{g}\right\rangle \mathbf{h}_{\mathbf{g}, j}
$$

\section{PRELIMINARIES}

Throughout this paper $\mathbb{H}$ denotes a separable Hilbert space. Given an index set $I$, a sequence $F=\left\{\mathbf{f}_{i}\right\}_{i \in I}$ of nonzero elements of $\mathbb{H}$ is a frame for $\mathbb{H}$, if there exist $0<A \leq B<\infty$ such that

$$
A\|\mathbf{f}\|^{2} \leq \sum_{i \in I}\left|\left\langle\mathbf{f}, \mathbf{f}_{i}\right\rangle\right|^{2} \leq B\|\mathbf{f}\|^{2} \quad \text { for all } \mathbf{f} \in \mathbb{H} .
$$

In finite dimensions, we find it useful to express frames as matrices, so we abuse the notation of $F$ as follows: when $\operatorname{dim} \mathbb{H}=$ $n$, a frame $F=\left\{\mathbf{f}_{i}\right\}_{i \in I}$ for $\mathbb{H}$ is often represented by a $n \times k$ matrix $F$, whose column vectors are $\mathbf{f}_{i}, 1 \leq i \leq k(k \geq n)$. The frame operator $S=F F^{*}$ is then positive, self-adjoint and invertible.

For each frame $F$ there exists at least one dual frame $G=$ $\left\{\mathbf{g}_{i}\right\}_{i \in I}$, satisfying

$$
\mathbf{f}=\sum_{i \in I}\left\langle\mathbf{f}, \mathbf{f}_{i}\right\rangle \mathbf{g}_{i}=\sum_{i \in I}\left\langle\mathbf{f}, \mathbf{g}_{i}\right\rangle \mathbf{f}_{i} \quad \text { for all } \mathbf{f} \in \mathbb{H} .
$$

The matrix equation $F G^{*}=G F^{*}=I$ is an equivalent expression to the frame representation (Equation 4). The set $\left\{\mathbf{g}_{i}=S^{-1} \mathbf{f}_{i}\right\}_{i \in I}$ is called the canonical dual frame.

Finding a dual frame can be computationally challenging; thus it is of interest to work with tight or scalable frames. We say that a frame is $A$-tight if $A=B$ in Equation (3). When $A=1$, we call $F$ a Parseval frame. If a frame $F=\left\{\mathbf{f}_{i}\right\}_{i \in I}$ is not tight, but we can find scaling coefficients $w_{i} \geq 0, i \in I$, such that the scaled frame $F_{w}=\left\{w_{i} \mathbf{f}_{i}\right\}_{i \in I}$ is tight, then we call the original frame $F$ a scalable frame. We note that the notion of scalability of a frame is defined for a unit-norm frame in [10], but in this manuscript we do not require a scalable frame to be unit-norm. If the scaling coefficients $w_{i}$ are positive for all $i \in I$, then we call the original frame $F$ a strictly scalable frame.

Let $I$ denote a finite or countable index set, let $G=\left\{\mathbf{f}_{s}\right\}_{s \in I} \subset \mathbb{H}$ and let $A: \mathbb{H} \rightarrow \mathbb{H}$ be a bounded operator. We call the collection

$$
F_{G}^{\mathrm{L}}(A)=\cup_{s \in I}\left\{A^{j} \mathbf{f}_{s}: j=0,1, \ldots, L_{s}\right\}
$$

a dynamical system, where $L_{s} \geq 0\left(L_{s}\right.$ may go to $\left.\infty\right)$ and $\mathbf{L}=$ $\left(L_{s}\right)_{s \in I}$ is a sequence of iterations related to the set $G$. The operator $A$, involved in generating the set Equation (5), is referred to as a dynamical operator. If $A$ is fixed, then we use the notation $F_{G}^{\mathbf{L}}$, and if $G=\{\mathbf{f}\}$ and $\mathbf{L}=\{L\}$, then we label (Equation 5) by $F_{\mathbf{f}}^{L}$.

Note that in Aldroubi et al. [1], $\mathbf{f}_{s}$ are chosen to be the standard basis vectors, while in this manuscript, we allow the use of any nonzero vector $\mathbf{f}_{s} \in \mathbb{H}$. If Equation (5) is a frame for $\mathbb{H}$, then we call $F_{G}^{\mathrm{L}}(A)$ a dynamical frame, generated by a dynamical operator $A$, set $G$ and sequence of iterations $\mathbf{L}$.

\section{NEW RESULTS ON DYNAMICAL FRAMES}

Theorem 1. Let $G=\left\{\mathbf{f}_{s}\right\}_{s \in I} \subset \mathbb{H}$, where $I$ is a countable index set, and assume that $F_{G}^{\mathrm{L}}(A)$ is a frame for $\mathbb{H}$, with frame operator $S$. The canonical dual frame of $F_{G}^{\mathrm{L}}(A)$ is the dynamical frame $F_{G^{\prime}}^{\mathbf{L}}(B)$, generated by operator $B=S^{-1} A S, G^{\prime}=\left\{\mathbf{g}_{s}=S^{-1} \mathbf{f}_{s}\right\}_{s \in I}$, and sequence of iterations $\mathbf{L}$.

In addition, every $\mathbf{f} \in \mathbb{H}$ can be reconstructed from the dynamical samples $\left\{\left\langle A^{* j} \mathbf{f}, \mathbf{f}_{s}\right\rangle \mid 0 \leq j \leq L_{s}\right\}_{s \in I}$ via the frame reconstruction formula

$$
\mathbf{f}=\sum_{s \in I} \sum_{j=0}^{L_{s}}\left\langle A^{* j} \mathbf{f}, \mathbf{f}_{s}\right\rangle B^{j} \mathbf{g}_{s}
$$

Proof: Let $F_{G}^{\mathrm{L}}(A)$ be a frame for $\mathbb{H}$, where $G=\left\{\mathbf{f}_{s}\right\}_{s \in I} \subset \mathbb{H}$ and $I$ is countable, and assume that $S$ is the frame operator for $F_{G}^{\mathbf{L}}(A)$. Then $S$ is an invertible operator, and for any $A^{j} \mathbf{f}_{s} \in F_{G}^{\mathbf{L}}(A)$ we have that $S^{-1}\left(A^{j} \mathbf{f}_{s}\right)$ is a canonical dual frame element, where $s \in I$, $0 \leq j \leq L_{s}$. If we choose

$$
\mathbf{g}_{s}:=S^{-1} \mathbf{f}_{s}, s \in I \text { and } B:=S^{-1} A S,
$$

then for all $j \geq 0$ we have

$$
B^{j} \mathbf{g}_{s}=\left(S^{-1} A S\right) \ldots\left(S^{-1} A S\right) \mathbf{g}_{s}=S^{-1} A^{j}\left(S \mathbf{g}_{s}\right)=S^{-1}\left(A^{j} \mathbf{f}_{s}\right)
$$

Theorem 2. Let $\mathbb{H}_{1}$ and $\mathbb{H}_{2}$ be two separable Hilbert spaces. Let $A$ be a bounded operator on $\mathbb{H}_{1}$ and let $B: \mathbb{H}_{1} \rightarrow \mathbb{H}_{2}$ be an invertible operator. Let I be a countable index set, and fix $G=\left\{\mathbf{f}_{s}\right\}_{s \in I} \subset \mathbb{H}_{1}$. Set $\mathbf{g}_{s}=B \mathbf{f}_{s} \in \mathbb{H}_{2}, s \in I$, and $C:=B A B^{-1}$. For any set of iterations $\mathbf{L}=\left(L_{s}\right)_{s \in I}, L_{s} \geq 0$, TFAE:

(i) The set $F=\cup_{s \in I}\left\{A^{j} \mathbf{f}_{s}\right\}_{j=0}^{L_{s}}$ is a frame for $\mathbb{H}_{1}$,

(ii) The set $B F=\cup_{s \in I}\left\{C^{j} \mathbf{g}_{s}\right\}_{j=0}^{L_{s}}$ is a frame for $\mathbb{H}_{2}$.

Proof: Let $\mathbf{g}_{s}=B \mathbf{f}_{s} \in \mathbb{H}_{2}, s \in I$, and set $C=B A B^{-1}$. Note that $C^{j}=B A^{j} B^{-1}$, due to $B^{-1} B=I$. For any $A^{j} \mathbf{f}_{s} \in F \subset \mathbb{H}_{1}$, we have

$$
B A^{j} \mathbf{f}_{s}=B A^{j} B^{-1} B \mathbf{f}_{s}=B A^{j} B^{-1} \mathbf{g}_{s}=C^{j} \mathbf{g}_{s} \in B F \subset \mathbb{H}_{2} .
$$

The operator $B$ is invertible, thus $B F$ is a frame if and only if $F$ is a frame, so (i) and (ii) are equivalent.

If the operator $B$ occurring in Theorem 2 is unitary and $\mathbb{H}_{1}=\mathbb{H}_{2}$, then the property of scalability is preserved. We have:

Corollary 1. Let $\mathbf{f}_{s} \in \mathbb{H}$, and set $\mathbf{v}_{s}=U^{*} \mathbf{f}_{s}$ for all $s \in I$, where $I$ is a countable index set. Let $A, R$ be two operators on a separable Hilbert space $\mathbb{H}$, and let $U$ be a unitary operator on $\mathbb{H}$. If $A=U R U^{*}$, then TFAE: 
(i) $\cup_{s \in I}\left\{A^{j} \mathbf{f}_{s}\right\}_{j=0}^{L_{s}}$ is a scalable frame for $\mathbb{H}$,

(ii) $\cup_{s \in I}\left\{R^{j} \mathbf{v}_{s}\right\}_{j=0}^{L_{s}}$ is a scalable frame for $\mathbb{H}$.

In the next section, we exploit the simplicity of the unitary diagonalization of normal operators to give more explicit conditions on the normal operator $A$ in order to ensure scalability of a frame of type $F_{G}^{\mathrm{L}}(A)$.

\subsection{Normal Operators}

Let $A$ be a bounded operator on a separable Hilbert space $\mathbb{H}$, and assume there exists a unitary operator $U$, and a diagonal operator $D$ such that $A=U D U^{*}$. Let $\mathcal{G}=\left\{\mathbf{f}_{s}\right\}_{s \in I}$ and set $\mathbf{v}_{s}=U^{*} \mathbf{f}_{s}, s \in I$, where $I$ is countable. Since $A^{j}=U D^{j} U^{*}$, it follows that for each $j \in \mathbb{N}_{0}$ we have

$$
A^{j} \mathbf{f}_{s}=U D^{j} U^{*} \mathbf{f}_{s}=U D^{j} \mathbf{v}_{s}=U\left(D^{j} \mathbf{v}_{s}\right) \text { for all } \mathbf{f}_{s} \in \mathcal{G} .
$$

By Corollary $1, \cup_{s \in I}\left\{A^{j} \mathbf{f}_{s}\right\}_{j=0}^{L_{s}}$ is a scalable frame for $\mathbb{H}$ if and only if $\cup_{s \in I}\left\{D^{j} \mathbf{v}_{s}\right\}_{j=0}^{L_{s}}$ is a scalable frame for $\mathbb{H}$.

By the spectral theorem, a compact self-adjoint operator on a Hilbert space can be unitarily diagonalized. Thus, we can deliver a more precise statement on dynamical frames scalability when a normal operator is involved; we note that the following result in the finite dimensional case can be generalized when working in a separable Hilbert space with a countable orthonormal basis:

Theorem 3. Let $A=U D U^{*}$ be a normal $n \times n$ matrix, where $U$ is unitary, and $D$ is diagonal, with diagonal entries $a_{1}, \ldots, a_{n} \in \mathbb{C}$. Let $\mathbf{f}_{s} \in \mathbb{H}$, and set $\mathbf{v}_{s}=U^{*} \mathbf{f}_{s}=\left(x_{s}(1), \ldots, x_{s}(n)\right)^{T}, 1 \leq s \leq p$.

The set $\cup_{s=1}^{p}\left\{A^{j} \mathbf{f}_{s} \mid 0 \leq j \leq L_{s}\right\}$ is a scalable frame of $\mathbb{H}$ if and only if there exists a positive solution $w_{s, 0}, w_{s, 1}, \ldots, w_{s, L_{s}}, 1 \leq s \leq p$ to the system of equations

$$
\left\{\begin{array}{l}
\sum_{s=1}^{p}\left|x_{s}(i)\right|^{2}\left[w_{s, 0}^{2}+w_{s, 1}^{2}\left|a_{i}\right|^{2}+\ldots+w_{s, L_{s}}^{2}\left|a_{i}\right|^{2 L_{s}}\right]=1, \\
\sum_{s=1}^{p} x_{s}(i) \bar{x}_{s}(j)\left[w_{s, 0}^{2}+w_{s, 1}^{2} a_{i} \bar{a}_{j}+\ldots+w_{s, L_{s}}^{2}\left(a_{i} \bar{a}_{j}\right)^{L_{k}}\right]=0,
\end{array}\right.
$$

for all $i, j=1, \ldots, n, i \neq j$.

Proof: Let $D$ be the unitary diagonalization of $A$, with diagonal entries $a_{1}, \ldots, a_{n} \in \mathbb{C}$, and let $\mathbf{v}_{s}=\left(x_{s}(1), \ldots, x_{s}(n)\right)^{T} \in \mathbb{C}^{n}$, $s \in\{1, \cdots, p\}, p \geq 1$.

The set $\cup_{s=1}^{p}\left\{D^{j} \mathbf{v}_{s} \mid 0 \leq j \leq L_{s}\right\}$ is a scalable frame for $\mathbb{H}$ if and only if there exist scaling coefficients $w_{s, 0}, w_{s, 1}, \ldots, w_{s, L_{s}}$, $1 \leq s \leq p$, which satisfy $\mathbf{f}=\sum_{s, j}\left\langle\mathbf{f}, w_{s, j} D^{j} \mathbf{v}_{s}\right\rangle w_{s, j} D^{j} \mathbf{v}_{s}$ for all $\mathbf{f} \in \mathbb{H}$. Restating the last equality in matrix product form illuminates the fact that $w_{s, j}^{2}$ need to be the positive solutions to the weighted system of Equation (11).

By Corollary 1 the conclusion of the theorem holds true for a finite dimensional Hilbert space $\mathbb{H}$.

Theorem 4. If $n \geq 3$, then a normal operator on $\mathbb{R}^{n}$ can not generate a strictly scalable dynamical frame from a single vector in $\mathbb{R}^{n}$.

Proof: The Equation (11) implies that for the first three $a_{i}^{\prime} s$, we always have the relation $a_{1} a_{2}, a_{1} a_{3}$, and $a_{2} a_{3}$ are all negative numbers assuming $w_{i} \neq 0, i=1,2,3$, which is not possible. Thus any diagonal operator for $\mathbb{R}^{n}$ can not generate a strictly scalable frame from a single vector in $\mathbb{R}^{n}$.

Although a normal operator on $\mathbb{R}^{n}$ can not generate a strictly scalable dynamical frame from a single vector, there do exist normal operators on $\mathbb{C}^{n}$ which generate scalable dynamical frames, for example harmonic tight frames.

The problem of finding specific conditions under which the set in item (ii) in Corollary 1 is a scalable frame for $\mathbb{H}$ is still open for operators which do not possess a unitary diagonalization. For this reason, we further study several operators with special structures, such as block-diagonal operators (section 4) and companion operators (section 5).

\section{BLOCK-DIAGONAL OPERATORS}

In this section, we explore the case when the operator $A$ is of block-diagonal form. Block-diagonal operators give us a chance to offer a partial answer to (Q1) in the case when we don't have a unitary diagonalization.

Let $A_{s}: \mathbb{H}_{s} \rightarrow \mathbb{H}_{s}$ be an operator on $\mathbb{H}_{s}$, with $\operatorname{dim} \mathbb{H}_{s}=n_{s}$, $1 \leq s \leq p$. Let $A: \mathbb{H} \rightarrow \mathbb{H}$ be a block-diagonal operator on $\mathbb{H}=\oplus_{s=1}^{p} \mathbb{H}_{s}$, constructed as follows:

$$
A=\left(\begin{array}{ccc}
A_{1} & \ldots & 0 \\
\vdots & \vdots & \vdots \\
0 & \ldots & A_{p}
\end{array}\right)
$$

Let $\mathbf{v} \in \mathbb{H}_{s}$ for some $1 \leq s \leq p$. If $\mathbb{H}$ is the direct sum of a family $\left(\mathbb{H}_{s}\right)_{s}$ of Hilbert space, for each index $s$ there exists a canonical inclusion $i_{s}$ of $\mathbb{H}_{s}$ into $H$. We say that $\mathbf{v}$ is well-embeded in $\mathbf{f} \in \mathbb{H}$ with respect to operator (Equation 12) if $\mathbf{f}=i_{s}(\mathbf{v})$.

Theorem 5. Let $A_{s}: \mathbb{H}_{S} \rightarrow \mathbb{H}_{s}$ be an operator on $\mathbb{H}_{s}$, with $\operatorname{dim} \mathbb{H}_{s}=n_{s}, 1 \leq s \leq p$. Let $A: \mathbb{H} \rightarrow \mathbb{H}$ be a block-diagonal operator on $\mathbb{H}=\oplus_{s=1}^{p} \mathbb{H}_{s}$, constructed as in Equation (12). Let $\mathbf{v}_{s, 1} \ldots, \mathbf{v}_{s, m_{s}} \in \mathbb{H}_{s}, 1 \leq s \leq p$ be well-embedded in $\mathbf{f}_{s, 1}, \ldots, \mathbf{f}_{s, m_{s}} \in$ $\mathbb{H}, 1 \leq s \leq p$.

$$
\text { The set } \bigcup_{s=1}^{p}\left\{A^{j} \mathbf{f}_{s, k} \mid 1 \leq k \leq m_{s}\right\}_{j=0}^{L_{s, k}}
$$

is a (scalable) frame of $\mathbb{H}$ if and only if $\left\{A_{s}^{j} \mathbf{v}_{s, k} \mid 1 \leq k \leq m_{s}\right\}_{j=0}^{L_{s, k}}$ are (scalable) frames of $\mathbb{H}_{s}$ for all $1 \leq s \leq p$.

Proof: We assume that all $m_{s}=1$, i.e., $\mathbf{f}_{s, k}=\mathbf{f}_{s}, \mathbf{v}_{s, k}=\mathbf{v}_{s}$, and $L_{s, k}=L_{s}, 1 \leq s \leq p$, to simplify the presentation of the proof. The matrix representation of $\cup_{s=1}^{p}\left\{A^{j} \mathbf{f}_{s}\right\}_{j=0}^{L_{s}}$ with scaling coefficients $w_{s, j}, 0 \leq j \leq L_{s}$ for each $s=1, \ldots, p$ is of block-diagonal form:

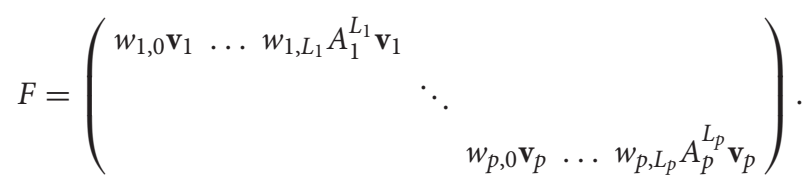


If $F$ is a tight frame, then row vectors of $F$ are orthogonal and have the same norm and so does $\left(w_{s, 0} \mathbf{v}_{s} \ldots w_{s, L_{s}} A_{s}^{L_{s}} \mathbf{v}_{s}\right)$ for each $s=1, \ldots, p$. This implies that the system $\left\{A_{s}^{j} \mathbf{v}_{s}\right\}_{j=0}^{L_{k}}$ is a scalable frame for $\mathbb{H}_{s}$ for all $1 \leq s \leq p$.

Now, suppose that for each $1 \leq s \leq p$, the system $\left\{A^{j} \mathbf{v}_{s}\right\}_{j=0}^{L_{s}}$ is a scalable frame for $\mathbb{H}_{s}$. Then, there exist some scaling coefficients $w_{s, j}, 1 \leq s \leq p, 0 \leq j \leq L_{s}$, such that $\left\{w_{s, j} A_{s}^{j} \mathbf{v}_{s} \mid 0 \leq j \leq L_{s}\right\}$ is a Parseval frame for each $s=1, \ldots p$.

Block operators we can employ in Theorem 5 include:

Example 1. Let $a, b, c, d$ be real numbers.

(a) The operator $A=\left(\begin{array}{ll}a & c \\ b & d\end{array}\right)$ in $\mathbb{R}^{2}$ generates a tight frame $F_{\mathbf{e}_{1}}^{2}$ if $a \neq-d, b=\frac{ \pm 1}{a+d} \sqrt{\frac{a^{2}(a+d)^{2}+(a+d)^{2}+a^{2}}{1+(a+d)^{2}}}$, and $c=$ $\mp a\left(a d+a^{2}+1\right) \sqrt{\frac{1+(a+d)^{2}}{(a+d)^{2}+a^{2}(a+d)^{2}+a^{2}}}$.

(b) The operator $A=\left(\begin{array}{ll}a & c \\ b & d\end{array}\right)$ in $\mathbb{R}^{2}$ generates a tight frame $F_{\mathbf{e}_{1}}^{2}$ if $a>0$ and $0<-\frac{a\left(a^{2}+b c\right)}{b^{2}(a+d)}<1$.

(c) The operator $A=\left(\begin{array}{ll}0 & a \\ 1 & b\end{array}\right)$ generates a strictly scalable frame $F_{\mathbf{e}_{1}}^{3}$ if $a+b^{2}<0$.

(d) The operator $A=\left(\begin{array}{lll}0 & 0 & 0 \\ 1 & a & c \\ 0 & b & d\end{array}\right)$ generates a strictly scalable frame $F_{\mathbf{e}_{1}}^{3}$ if $a>0$ and $0<-\frac{a\left(a^{2}+b c\right)}{b^{2}(a+d)}<1$.

The conditions for Example 1(b) are obtained from the following proposition:

Proposition 1. Let $a, b, c, d$ be real numbers such that $a>0$ and $a b c d \neq 0$. Then the following two statements are equivalent:

(1) $0<-\frac{a c}{b d}<1$.

(2) The system

$$
F=\left(\begin{array}{lll}
1 & a & c \\
0 & b & d
\end{array}\right)
$$

is a strictly scalable frame for $\mathbb{R}^{2}$.

Proof: We first note that the condition $0<-\frac{a c}{b d}<1$ is equivalent to $\left(a>0,-\frac{b}{c}>\frac{a}{d}>0\right)$ or $\left(a>0,-\frac{d}{a}>\frac{c}{b}>0\right)$. $(1) \Rightarrow(2): \quad$ The conditions $a>0,-\frac{b}{c}>\frac{a}{d}>0$ imply that

$$
d>0, a d-b c>0, \frac{a c}{b d}>-1
$$

and the conditions $a>0,-\frac{d}{a}>\frac{c}{b}>0$ imply that

$$
d<0, a d-b c<0, \frac{a c}{b d}>-1 .
$$

Then

$$
x=\sqrt{\frac{a c}{b d}+1}, y=\sqrt{\frac{c}{-b(a d-b c)}}, z=\sqrt{\frac{a}{d(a d-b c)}}
$$

are positive numbers and

$$
F=\left(\begin{array}{ccc}
x & y a & z c \\
0 & y b & z d
\end{array}\right)
$$

is a Parseval frame for $\mathbb{R}^{2}$.

$(1) \Leftarrow(2)$ : If the system $F$ is strictly scalable, then the normalized system

$$
F^{\prime}=\left(\begin{array}{ccc}
1 & \frac{a}{\sqrt{a^{2}+b^{2}}} & \frac{c}{\sqrt{c^{2}+d^{2}}} \\
0 & \frac{b}{\sqrt{a^{2}+b^{2}}} & \frac{d}{\sqrt{c^{2}+d^{2}}}
\end{array}\right)
$$

is a unit-norm scalable frame. The Gramian matrix of diagram vectors of $F^{\prime}$ has positive scalings in its null space:

$$
\begin{gathered}
\frac{a^{2} c d-a b c^{2}+a b d^{2}-b^{2} c d}{a b\left(c^{2}+d^{2}\right)}>0, \\
\frac{-c d\left(a^{2}+b^{2}\right)}{a b\left(c^{2}+d^{2}\right)}>0 .
\end{gathered}
$$

Inequality (Equation 15) implies that $-\frac{a c}{b d}>0$. Next we show that $-\frac{a c}{b d}<1$.

In case $b>0$, inequality (Equation 14) implies that

$$
a^{2} c d+a b d^{2}>b c(a c+b d)
$$

If $(c>0$ and $a c+b d \geq 0)$ or $(c<0$ and $a c+b d \leq 0)$, then $a^{2} c d+a b d^{2}>0$, which implies $-\frac{a c}{b d}<1$. If $c>0$ and $a c+b d<0$, then $a c<-b d$, which implies $1<-\frac{b d}{a c}$ since $a c>0$. Similarly, if $c<0$ and $a c+b d>0$, then $a c>-b d$, which implies $1<-\frac{b d}{a c}$ since $a c<0$. This is equivalent to $-\frac{a c}{b d}<1$. In case $b<0$, suppose that $-\frac{a c}{b d} \geq 1$. Multiply both sides by the positive number $-a b d^{2}$. On one hand we have $a^{2} c d \geq$ $-a b d^{2}$ and on the other hand, from inequality (14), we have $a^{2} c d-a b c^{2}<-a b d^{2}+b^{2} c d$. Since $a^{2} c d \geq-a b d^{2}$, we have $-a b d^{2}-a b c^{2}<-a b d^{2}+b^{2} c d$, which implies $-\frac{a c}{b d}<1$. This contradicts our assumption.

Proposition 2. Let $i, j, k, l \in \mathbb{N}$ be such that $p<k \leq n, q<$ $l \leq n$, and let $N \in \mathbb{N}$. For each $m=1, \ldots, N$, we define $A_{k l}^{p \bar{q}}(m)=\left[a_{i j}(m)\right]_{i, j=1}^{n}$ as

$$
a_{p q}(m)=a_{m}, a_{p l}(m)=b_{m}, a_{k q}(m)=c_{m}, a_{k l}(m)=d_{m}
$$

If for each $m=1, \ldots, N, a_{m}, b_{m}, c_{m}$ and $d_{m}$ satisfy the conditions of Proposition 1, and the system

$$
\left\{\mathbf{e}_{1}\right\} \cup \cup_{m=1}^{N}\left\{A_{k l}^{p q}(m) \mathbf{e}_{1},\left(A_{k l}^{p q}(m)\right)^{2} \mathbf{e}_{1}\right\}
$$

spans $\mathbb{R}^{n}$, then Equation (16) is a strictly scalable frame for $\mathbb{R}^{n}$. 


\subsection{Generalization}

The problem of generating a frame by iterative actions of a blockdiagonal operator (Equation 12) is equivalent to the problem of generating a frame by iterative actions of multiple operators. So, we pose the question: Given operators $A_{s}, s \in \mathbb{N}$, on a separable Hilbert space $\mathbb{H}$, and fixed vectors $\mathbf{v} \in G \subset$ $\mathbb{H}$, when is the collection $\left\{A_{s} \mathbf{v} \mid s \in \mathbb{N}, \mathbf{v} \in G\right\}$ a (scalable) frame for $\mathbb{H}$ ?

Such frames would naturally arise in applications; for instance, let $\mathbf{f} \in \mathbb{H}$ be the initial state of a physical system that evolves through time, and let the sequence of bounded operators $\left\{A_{s}^{*} \mid s \in\right.$ $\mathbb{N}\}$ describe the evolution of that physical system i.e., $\mathbf{f}_{s}=A_{s}^{*} \mathbf{f}$ is the $s$ th state of the system at time $t_{s}$. Assume there are several fixed sampling locations $\mathbf{v} \in G \subseteq \mathbb{H}$, where samples of the evolved state are taken:

$$
\left\langle A_{s}^{*} \mathbf{f}, \mathbf{v}\right\rangle=\left\langle\mathbf{f}, A_{s} \mathbf{v}\right\rangle .
$$

Clearly, $\mathbf{f}$ can be recovered from the set of samples $\left\{\left\langle A_{s}^{*} \mathbf{f}, \mathbf{v}\right\rangle \mid \mathbf{v} \in\right.$ $G\}$ if and only if $\left\{A_{s} \mathbf{v} \mid \mathbf{v} \in G, s \in \mathbb{N}\right\}$ is a frame for $\mathbb{H}$. Note that this type of a frame is a generalization of frames of iterative actions of operators, when one or more operators are involved.

For a frame generated by actions of a sequence of operators, we find that its canonical dual frame has similar structure:

Theorem 6. Let $A_{s}, s \in I$ be a sequence of operators on a separable Hilbert space $\mathbb{H}$, and let $G \subset \mathbb{H}$. Suppose $F\left(A_{s}, I\right):=\left\{A_{s} \mathbf{v} \mid s \in\right.$ $I, \mathbf{v} \in G\}$ is a frame for $\mathbb{H}$, with frame operator $S$. Then its canonical dual frame is

$$
\left\{B_{s} \mathbf{f} \mid s \in I, \mathbf{f} \in S^{-1}(G)\right\} \text {, where } B_{s}=S^{-1} A_{s} S, s \in I .
$$

Proof: If $S$ denotes the frame operator of the frame $F\left(A_{s}, I\right)$ for $\mathbb{H}$, then its canonical dual frame elements are $S^{-1} A_{s} \mathbf{v}$, $\mathbf{v} \in G$.

Since $S S^{-1}$ is the identity operator, and $B_{s}=S^{-1} A_{s} S$, we obtain that the dual frame elements are

$$
S^{-1} A_{s} \mathbf{v}=S^{-1} A_{s} S S^{-1} \mathbf{v}=S^{-1} A_{s} S \mathbf{f}=B_{s} \mathbf{f} \text {, where } \mathbf{f} \in S^{-1}(G) .
$$

\section{COMPANION OPERATORS AND GENERALIZATIONS}

Let $a_{1}, \ldots, a_{n} \in \mathbb{R}$ which are not all zeros; then

$$
A=\left(\begin{array}{c|c}
0 & a_{1} \\
\hline & a_{2} \\
I_{n-1} & \vdots \\
& a_{n}
\end{array}\right)
$$

is called a companion operator [14].

Proposition 3. Let the dynamical operator $A$ be a companion operator (18) in $\mathbb{R}^{n}$, then we have
(1) $F_{\mathbf{e}_{1}}^{n-1}=I$.

(2) for any orthogonal matrix $U$, the operator $U A U^{-1}$ generates an orthonormal basis $U$.

It is known that the standard orthonormal basis $B$ can not be extended to a scalable frame by adding one vector $\mathbf{f} \in \mathbb{H} \backslash B$, [8, 11]. Thus, we explore when one can generate a dynamical frame by adding two vectors. Although a companion operator $A$ does not generate a scalable frame $F_{\mathbf{e}_{1}}^{n}$, it can generate a scalable frame $F_{\mathbf{e}_{1}}^{n+1}$ under certain conditions. Using the companion operator $A$, we have

$$
F_{\mathbf{e}_{1}}^{n}=\left(\mathbf{e}_{1} \ldots \mathbf{e}_{n} \mathbf{f}\right), \quad F_{\mathbf{e}_{1}}^{n+1}=\left(\mathbf{e}_{1} \ldots \mathbf{e}_{n} \mathbf{f} \mathbf{g}\right),
$$

where

$$
\mathbf{f}=\left(\begin{array}{c}
a_{1} \\
a_{2} \\
a_{3} \\
\vdots \\
a_{n-1} \\
a_{n}
\end{array}\right) \text { and } \mathbf{g}=\left(\begin{array}{c}
a_{1} a_{n} \\
a_{1}+a_{2} a_{n} \\
a_{2}+a_{3} a_{n} \\
\vdots \\
a_{n-2}+a_{n-1} a_{n} \\
a_{n-1}+a_{n}^{2}
\end{array}\right) \text {. }
$$

Proposition 4. [11] Let $\left\{\mathbf{e}_{1}, \ldots \mathbf{e}_{n}\right\}$ be the standard orthonormal basis in $\mathbb{R}^{n}$ with $n \geq 2$. Let $\mathbf{f}$ and $\mathbf{g}$ be two unit-norm vectors in $\mathbb{R}^{n}$.

If either system $\left\{\mathbf{e}_{1}, \ldots \mathbf{e}_{n}, \mathbf{f}, \mathbf{g}\right\}$ or $\left\{\mathbf{e}_{1}, \ldots \mathbf{e}_{n-1}, \mathbf{f}, \mathbf{g}\right\}$ is scalable, then $\mathbf{f}$ and $\mathbf{g}$ have only two nonzero elements in the same entries.

We now assume that $F_{\mathbf{e}_{1}}^{n+1}$ is scalable. Then by Proposition 4, $a_{m}=0$ implies that $a_{m-1}=0$ for $m \geq 2$. This implies that $a_{1}=\ldots=a_{n-2}=0$. A consequence of this is the following result.

Proposition 5. Let $a$ and $b$ be real numbers such that $a>0$ and $0<-\frac{a^{2}}{a+b^{2}}<1$. Then the companion operator $A$ in $\mathbb{R}^{n}$,

$$
A=\left(\begin{array}{cccccc}
0 & 0 & \ldots & 0 & 0 & 0 \\
1 & 0 & \ldots & 0 & 0 & 0 \\
& . & . & . & . & \\
0 & 0 & \ldots & 1 & 0 & a \\
0 & 0 & \ldots & 0 & 1 & b
\end{array}\right)
$$

generates a strictly scalable frame $F_{\mathbf{e}_{1}}^{n+1}$.

Proof: We have

$$
F_{\mathbf{e}_{1}}^{n+1}=\left(\begin{array}{ccccc}
I_{n-2} & & & & \\
& 1 & 0 & a & a b \\
& 0 & 1 & b & a+b^{2}
\end{array}\right)
$$

which is strictly scalable.

We note that the operator $A$ in Equation (20) is not diagonalizable. 
Example 2. Let $a$ and $b$ be real numbers such that $0<$ $-\frac{a\left(a^{2}+b c\right)}{b^{2}(a+d)}<1$ and $a>0$. Then the operator

$$
A=\left(\begin{array}{ccccc}
0 & 0 & 0 & \ldots & 0 \\
1 & 0 & 0 & \ldots & 0 \\
0 & 1 & 0 & \ldots & 0 \\
\vdots & \vdots & \vdots & \vdots & \\
0 & \ldots & 1 & a & c \\
0 & \ldots & 0 & b & d
\end{array}\right)
$$

generates a strictly scalable frame $F_{\mathbf{e}_{1}}^{n}$ for $\mathbb{R}^{n}$.

Proof: We have

$$
F_{\mathbf{e}_{1}}^{n}=\left(\begin{array}{cccc}
I_{n-2} & & & \\
& 1 & a & a^{2}+b c \\
& 0 & b & a b+b d
\end{array}\right) .
$$

The strict scalability follows by Example 1 .

Example 3. Let $2 \sin ^{2}(\phi)-1>0$ and let

$$
A=\left(\begin{array}{cccccc} 
\pm 1 & 0 & 0 & 0 & \ldots & 0 \\
0 & \pm 1 & 0 & 0 & \ldots & 0 \\
\vdots & \vdots & \vdots & \vdots & \vdots & \vdots \\
0 & 0 & \ldots & \pm 1 & 0 & 0 \\
0 & 0 & \ldots & 0 & \cos \phi & -\sin \phi \\
0 & 0 & \ldots & 0 & \sin \phi & \cos \phi
\end{array}\right)
$$

The set

$$
\left\{\mathbf{e}_{n-1}, A \mathbf{e}_{n-1}, A^{2} \mathbf{e}_{n-1}\right\} \cup \bigcup_{l=1}^{n-2}\left\{\mathbf{e}_{l}, A \mathbf{e}_{l}, \ldots, A^{L_{l}} \mathbf{e}_{l}\right\}
$$

is a strictly scalable frame of $\mathbb{R}^{n}$.

\section{CONCLUDING REMARKS}

We have studied the scalability of dynamical frames in a separable Hilbert space $\mathbb{H}$. Given an operator $A$ on $\mathbb{H}$ and a (at most countable) set $G \subset \mathbb{H}$, we have explored the relations between $A$, $G$ and the number of iterations that make the system (1) a scalable frame. In section 3.1 we have fully answered question (Q1) in finite dimensions, when working with a normal operator. We have also studied operators with specialized structures, such as block-diagonal operators (section 4), and companion operators (section 5), which are not necessarily normal; for instance, note that the block-diagonal matrix $A$ in Theorem 5 cannot be normal if one of its blocks is not normal.

In adition, we have established the canonical dual frame for frames of type $F_{G}(A)$; in particular, we showed that the canonical dual frame has, as anticipated, an iterative set structure; in section 4.1, we had generalized the notion of dynamical frames to the notion of frames generated by sequences of operators, and verified that the canonical dual frame result is generalized as well (Theorem 6); this type of result holds true in any separable Hilbert space $\mathbb{H}$.

\section{AUTHOR CONTRIBUTIONS}

All authors listed, have made substantial, direct and intellectual contribution to the work, and approved it for publication.

\section{FUNDING}

YK was supported by the Central Michigan University FRCE Research Type A Grant \#C48143. RA was supported by the Ball State University Aspire Research Award \#I601-16.

\section{ACKNOWLEDGMENTS}

We express our gratitude to Professor S. Narayan for many helpful conversations on this work.

Fourier Anal Appl. (2015) 21:11-31. doi: 10.1007/s00041-0149359-9

6. Aldroubi A, Davis J, Krishtal I. Dynamical sampling: time space trade-Off. Appl Comput Harmon Anal. (2013) 34:495-503. doi: 10.1016/j.acha.2012. 09.002

7. Cahill J, Chen X. A note on scalable frames. In: Proceedings of the 10th International Conference on Sampling Theory and Applications, Bremen (2013). p. 93-96.

8. Kutyniok G, Okoudjou K, Philipp F. Scalable frames and convex geometry. Contemp Math. (2013) 626:19-32. doi: 10.1090/conm/626/ 12507

9. Kutyniok G, Okoudjou KA, Philipp F, Tuley EK. Scalable frames. Linear Algebra Appl. (2013) 438:2225-38. doi: 10.1016/j.laa.2012. 10.046

10. Copenhaver M, Kim Y, Logan C, Mayfield K, Narayan SK, Petro MJ, Sheperd J. Diagram vectors and tight frame scaling in finite dimensions. Oper Matrices (2014) 8:78-88. doi: 10.7153/oam08-02

11. Domagalski R, Kim Y, Narayan SK. On minimal scalings of scalable frames. In: Proceedings of the 11th International Conference on Sampling Theory and 
Applications, Washington, DC (2015). p. 91-95. doi: 10.1109/SAMPTA.2015. 7148857

12. Chen X, Kutyniok G, Okoudjou KA, Philipp F, Wang R. Measures of scalability. IEEE Trans Inf Theory (2015) 61:4410-23. doi: 10.1109/TIT.2015.2441071

13. Benedetto JJ, Fickus M. Finite normalized tight frames. $A d v$ Comput Math. (2003) 18:357-85. doi: 10.1023/A:1021323 312367

14. Horn RA, Johnson CR. Matrix Analysis. Cambridge: Cambridge University Press (2012). doi: 10.1017/CBO9781139020411
Conflict of Interest Statement: The authors declare that the research was conducted in the absence of any commercial or financial relationships that could be construed as a potential conflict of interest

Copyright (C) 2017 Aceska and Kim. This is an open-access article distributed under the terms of the Creative Commons Attribution License (CC BY). The use, distribution or reproduction in other forums is permitted, provided the original author(s) or licensor are credited and that the original publication in this journal is cited, in accordance with accepted academic practice. No use, distribution or reproduction is permitted which does not comply with these terms. 